\title{
An Opportunistic Maintenance Policy of Multi-unit Series Production System with Consideration of Imperfect Maintenance
}

\author{
Wenrui Hou ${ }^{1}$ and Zuhua Jiang ${ }^{2}$ \\ ${ }^{1}$ Department of Industrial Engineering, School of Mechanical Engineering, Shanghai Jiao Tong University, Shanghai 200240 P. R. \\ China \\ ${ }^{2}$ Department of Industrial Engineering, School of Mechanical Engineering, Shanghai Jiao Tong University, Shanghai 200240 P. R. \\ China
}

\begin{abstract}
This paper proposes an opportunistic maintenance policy for the multi-unit series production system. This proposed maintenance policy is on the basis of the failure rate analysis, which is more realistic than age-based or time-based opportunistic replacement models. When a system halts for performing preventive maintenance or opportunistic maintenance, the alternative decision of replacement and repair is made by economic evaluation of these two types of maintenance actions. The optimal preventive maintenance plans in a finite time horizon can be obtained by adjusting the preventive maintenance threshold and opportunistic maintenance threshold. A numerical example is given for illustrating the applications of the proposed policy.
\end{abstract}

Keywords: Multi-unit series production system; Opportunistic maintenance; Failure rate; Imperfect maintenance; Economic evaluation

\section{Introduction}

Production systems usually deteriorate with usage and time. During the process of production, manufacturers need to avoid failure, which increases total production cost and also incurs the loss of raw materials. It has been proven that the probability of system unscheduled breakdown can be reduced effectively by preventive maintenance. Thus an appropriate preventive maintenance policy is very important for production systems. There are plenty of literatures on the preventive maintenance for single-unit system. Nowadays, more and more researchers have been focusing on models of multi-unit series production system maintenance.

Opportunistic maintenance means that a unit will perform preventive maintenance only when its maintenance opportunity reaches some certain value. Opportunistic maintenance model was firstly presented by Radner and Jorgenson(1963)[1]. They proposed a model of one component replacement in the presence of several monitored parts. Some extensions of opportunistic maintenance were presented afterwards. Berg (1976, 1978) suggested an opportunistic maintenance model for two identical components subject to exponential failure, in that model both components are replaced either when one of them fails and the other's age exceeds a predetermined threshold[2,3]. Dekker and Smeitink(1991) suggested an opportunity-based block replacement model, in which the minimized expected average maintenance cost can be obtained for an infinite time horizon[4]. Dekker and Kijkstra(1992) gave an opportunity-based components replacement policy, in which preventive replacements are only possible at other components' maintenance opportunities, and the opportunities occur according to a Poison process[5].

The opportunistic maintenance has been proven that there are many situations in which opportunistic maintenance is an effective way to improve system reliability, decrease the random failures frequency of the system. For example, when a system halts for performing preventive maintenance on some units, others units are

\footnotetext{
*Corresponding author e-mail: houwr@163.com
} 
being taken some opportunistic maintenance, which may be cost-efficient. Zheng and Fard (1991) presented a hazard rate-based opportunistic maintenance model for the system with multiple different units, in which the decision of alternative of repair and replacement is made according to the hazard rate and hazard rate tolerance[6]. Stinson and Khumawala (1987) developed a heuristic procedure to solve the problem of planning machine replacements for a serially dependent production system in a given finite planning horizon, and based which Sarkis, J. and Semple, J. (1994) presented a new reformulation to simplify the model constraint and integer variables and prove the advantage[7,8].

The classical opportunistic-maintenance policies are almost age-based or time-dependent, in which a unit of system is undertaken maintenance at a fixed age or a certain time. Zheng (1995) extended the model of Zheng and Fard (1991) and an all opportunity-triggered age-depended replacement policy for multiple-unit systems was presented, in which opportunities are created by unit replacements when its hazard rate is within the predetermined tolerance or unit random failures before replacement[9]. Wijnmalen and Hontelez(1996) presented a general multi-component system opportunistic repair model, which is a natural generalization of the opportunistic maintenance model introduced by $\operatorname{Berg}(1978)$ for two identical components system, in which upper and lower control limits for the repair of components are optimal objects to minimize long-run average cost per unit of time for the whole system[10]. a stochastic model was developed by M.Sqvsar, which describes the states of a manufacturing system by a set of differential equations [11]. Pham and Wang(2000) proposed two age-based opportunistic maintenance policies for a system, in which minimal repair was introduced, and Pham and Wang(2000) further extended these two policies to the one with the consideration of the number of failed components[12].

In practice there are some problems, for example, the unit state deterioration process is not always linear with the age or service time, and usually affect the maintenance cost and the unit functioning duration. Another point is that the selection of maintenance actions is almost based on the age in previous literatures, which ignored the relationship between effectiveness and cost of maintenance actions. Rao and Bhdury (2000) studied a new opportunistic maintenance policy based on the classification of opportunities for multi-system systems, in which each system of system consists of many components or subsystems connected in series[13]. Satow and Osaki(2003) presented an opportunity-based age replacement policy with different intensity rates, which extended the classical opportunity-based maintenance model that supposed the opportunities are generated according to a homogeneous Poisson process[14]. Zhou and $\mathrm{Xi}$ (2006) developed a dynamic opportunistic maintenance policy for a continuously monitored series system with integrating imperfect effect into maintenance activities based on short-time information of the system, and the optimization was obtained to maximize the short-term cumulative opportunity maintenance cost-saving[15]. Levrat and Thomas (2008) proposed a new approach for integrating opportunistic maintenance into production planning, which uses the 'odds algorithm' and is based upon the theory of optimal stopping for mono-component or multi-component systems[16]. Xiaojun Zhou and Lifeng Xi(2009,2010)proposes an opportunistic PM scheduling algorithm for the multi-unit series system based on dynamic programming with the integration of the imperfect effect into maintenance actions and optimal maintenance practice is determined by maximizing the short-term cumulative opportunistic maintenance cost savings for the whole system[17,18]. Tim Bedford and Isha Dewan (2011)present a competing risks reliability model for a system, in which the released signals are used to inform opportunistic maintenance[19].

In this paper, a new opportunistic maintenance policy is proposed for multi-unit repairable systems with consideration of nonlinear deterioration process with age and introduction of imperfect maintenance. The failure rate functions of units of the system are used to describe the unit state. The system state is deteriorating with the increasing failure rate of the units. The failure rate and maintenance cost work together as the thresholds of preventive maintenance and opportunistic maintenance. Three types of maintenance actions, including replacement, repair and minimal repair, are introduced into the opportunistic maintenance model to make it more in line with the actual situations. The maintenance is classified by the maintenance effect. Replacement is used to restore the unit to a "good-as-new" condition, which is also called perfect maintenance; minimal repair is used to restore the unit to a "as bad as old" condition, which was presented in Barlow and Hunter(1960)[20]. Later, the models about minimal repair and imperfect maintenance were established by Pham and Wang (1996)[21]; and repair is used to restore the unit state somewhere between "as good as new" and "as bad as old". It is also called imperfect maintenance. The effective age renewal factor is introduced to describe the reduction of failure rate of unit if it is performed repair.

The purpose of this paper is to present an opportunistic maintenance model for Multi-unit series production system with consideration of nonlinear deterioration process with age or time, and introduction of more maintenance actions to make the model to be more line in actual. It can minimize the expected total maintenance cost (including preventive maintenance and corrective maintenance), and at same time, ensures the expected system total maintenance cost and downtime losses to be below a specified level, for each unit of the system at any time in a finite time horizon. 


\section{Definition and preliminaries}

Consider a system with $M(M \geq 2)$ failure rate independent units connected in series, the failure rate function $\lambda_{i}(t)$ is used to be the index of the state of unit $i(i=1, \ldots, M)$. The value $E^{p}$ is of the expected total maintenance cost and $E^{o}$ is of and the system scheduled downtime losses. $E^{p}$ and $E^{o}$ are used to be the thresholds of preventive maintenance and opportunistic maintenance respectively. $E_{i}(t)$ is of the expected scheduled maintenance cost and downtime losses of unit $i$,it can be obtained by its failure rate at $t$ and the scheduled maintenance cost and downtime losses. If $E_{i}(t)$ reaches $E^{p}$, then the system will be halted to be performed preventive maintenance on unit $i$, at same time, the maintenance opportunity is created for other units, if $E^{o} \leq E_{k}(t) \leq E^{p}(k=1, \ldots, M \mathrm{M} k \neq i)$. Then the scheduled downtime losses of unit $k$ will not be cumulated, which means the money is saved.

If unit $i$ will be performed preventive maintenance or opportunistic maintenance, there are two types of maintenance actions can be chosen: replacement or repair. In this paper, the more efficient one will be selected. When some units which need to be taken preventive maintenance, how to make decision to select more economical maintenance action are important. In this paper, the decision is made through the expected failure rate reduced quantum per unit cost of each type of maintenance actions. The expected failure rate reduced quantum per unit cost of unit $i$ at $t_{j}$ is represented by $\rho_{i, j}$.

Once unscheduled breakdown occurs before replacement or repair, the corrective maintenance will be performed in time, and the other units' opportunistic maintenance will not be preformed. This is because in practice the maintenance resources such as spare parts, engineers maybe not are prepared for taking the opportunistic maintenance activities. When there are some random failures, minimal repair will be taken on the failure units and will recover the unit states only, but will not change their failure rates.

When the finite time horizon $[0, T]$ is given, the maintenance plan can be obtained by aforementioned policy, and the optimal total maintenance cost can be obtained by adjusting the maintenance thresholds $E^{o}$ and $E^{p}$.

\section{Definition}

Cycle: the time interval between two scheduled halts, for example, the $j$ th cycle is the time interval $\left[t_{j-1}, t_{j}\right]$ between $(j-1)$ th and $j$ th scheduled halt.

\section{Notation \\ Assumptions}

(1) The maintenance actions take negligible time.

(2) Technique and resources exist to correct all failed units by minimal repair before being taken preventive or opportunistic maintenance.

(3) The system runs continuously except being performed maintenance.

\begin{tabular}{|c|c|}
\hline$t_{j}$ & $\begin{array}{l}\text { time to perform preventive maintenance when the } \\
j \text { th system scheduled halt }\end{array}$ \\
\hline$\lambda_{i}(t)$ & failure rate function of unit $i$ \\
\hline$\lambda_{i, j}(t)$ & failure rate function of unit $i$ in the $j$ th Cycle \\
\hline$e_{i, j}$ & effective age of unit $i$ before being maintained at $t_{j}$ \\
\hline$e_{i, j}^{\prime}$ & effective age of unit $i$ after being maintained at $t_{j}$ \\
\hline & effective age renewal factor of repair \\
\hline$v_{i}$ & value or price of unit $i$ \\
\hline$d_{i, j}$ & $\begin{array}{l}\text { duration of unit } i \text { functioning without replacement } \\
\text { and repair till the } j \text { th scheduled halt }\end{array}$ \\
\hline$c_{i}^{p}$ & cost of replacement action of unit $i$ \\
\hline$c_{i}^{r}$ & cost of repair action of unit $i$ \\
\hline$c_{i}^{c}$ & cost of minimal repair action of unit $i$ \\
\hline$l^{s}$ & downtime losses of the scheduled halt \\
\hline$l^{u}$ & $\begin{array}{l}\text { downtime losses of the unscheduled breakdown } \\
\text { expected failure rate reduced quantum per unit } i\end{array}$ \\
\hline$\rho_{i, j}^{p p} \rho_{i, j}^{p r}$ & $\begin{array}{l}\text { cost of unit by replacement and repair if it is taken } \\
\text { preventive maintenance at } t_{j} \\
\text { expected failure rate reduced quantum per unit } i\end{array}$ \\
\hline$\rho_{i, j}^{o p} \rho_{i, j}^{o r}$ & $\begin{array}{l}\text { cost of unit by replacement and repair if it is taken } \\
\text { opportunistic maintenance at } t_{j}\end{array}$ \\
\hline
\end{tabular}

(4) At the initial instant, the state of each unit is completely new.

(5) There are not any buffers between the units of system.

(6) The failure rate function of each unit is continuous and strictly increasing in each preventive maintenance cycle.

(7) The cost of corrective maintenance is higher than the repair, and the repair is higher than replacement for each unit.

Please note that according to assumption 3, is usually less than because if then the units will never be performed opportunistic maintenance, thus the maintenance opportunities will never be triggered.

\section{Mathematical model}

\subsection{Maintenance cost}

In a given finite time horizon $[0, T]$, consider a series system with $M(M \geq 2)$ units that are subject to $N(N>0)$ times scheduled halts to be performed preventive maintenance and opportunistic maintenance activities. The total maintenance cost of the system includes preventive maintenance cost, opportunistic maintenance cost, corrective maintenance cost, and losses of scheduled and unscheduled downtime, which can be represented by:

$$
C(T)=C^{t p}(T)+C^{t c}(T)
$$

where $C^{t p}(T)$ include the expected total cost of $N$ times preventive maintenance activities and the downtime losses of scheduled halts, and $C^{t c}(T)$ includes the expected total cost of corrective maintenance and the 
downtime losses of unscheduled breakdown in the time horizon $[0, \mathrm{~T}]$.

The total cost of $N$ times preventive maintenance activities is given by:

$$
C^{t p}(T)=\sum_{j=1}^{N} C_{j}^{p}\left(t_{j}\right)=\sum_{j=1}^{N} \sum_{i=1}^{M} c_{i, j}\left(t_{j}\right)
$$

Where $C_{j}^{p}\left(t_{j}\right)$ is the sum of the expected total maintenance cost and the scheduled downtime losses of $M$ units at $t_{j}, c_{i, j}\left(t_{j}\right)$ is the sum of the expected total maintenance cost and the scheduled downtime losses of unit $i$ at $t_{j}, i=1, \ldots, M, j=1, \ldots, N$. At $t_{j}$, if $c_{i, j}\left(t_{j}\right)$ of unit $i$ reaches the threshold of preventive maintenance $E^{P}$, then preventive maintenance will be taken on unit $i$, at the same time, the maintenance opportunity is created for other units.

According to the relationship between $E_{i}\left(t_{j}\right)$ and the thresholds of maintenance of unit $i$, it is determined whether unit $i$ will be performed maintenance and the type of maintenance actions performed on unit at $t_{j} . E_{i}\left(t_{j}\right)$ is given by:

$$
E_{i}\left(t_{j}\right)=\left(c_{i}^{c}+l^{u}\right) \lambda_{i, j}\left(t_{j}\right)
$$

And then, $c_{i, j}\left(t_{j}\right)$ can be obtained by:

$$
c_{i, j}\left(t_{j}\right)=\left\{\begin{array}{l}
v_{i} \exp \left(-\int_{0}^{e_{i, j}} \lambda_{i}(t) d t\right)+c_{i}^{p}+l^{s}, E_{i}\left(t_{j}\right) \geq E^{p} \\
\text { (preventive maintenance,replacement) } \\
c_{l}^{r}+l^{s}, E_{i}\left(t_{j}\right) \geq E^{p} \\
(\text { preventivemaintenance, repair) } \\
v_{i} \exp \left(-\int_{0}^{e_{i, j}} \lambda_{i}(t) d t\right)+c^{p}, E^{P}>E_{i}\left(t_{j}\right) \geq E^{o} \\
\text { (opportunistic maintenance,replancement) } \\
c_{l}^{r}, E^{p}>E_{i}\left(t_{j}\right) \geq E^{o} \\
\text { (opportunistic maintenance,repair) } \\
0, E_{i}\left(t_{j}\right)<E^{o} \\
(\text { no maintenance) }
\end{array}\right.
$$

If unit $i$ is still functioning but replaced by a new one at $t_{j}, i=1, \ldots, M, j=1, \ldots, N$, then $v_{i} \exp \left(-\int_{0}^{e_{i, j}} \lambda_{i}(t) d t\right)$ is the waste value. For the units are taken opportunity maintenance, $c_{i, j}\left(t_{j}\right)$ are not be reckoned in the downtime losses resulting from the scheduled halts, because which is calculated in the one of preventive maintenance. How to make the decision of the two alternative types of maintenance actions (replacement and repair) will be given later on.

Prior to the state indices of units reach the maintenance threshold, their states are comparatively "young", repair or replacement is not necessary for them. Therefore, minimal repair, which takes small amount of money and other maintenance resources, will be taken on them. In (1), $C^{t c}(T)$ include: corrective maintenance cost, losses of unscheduled breakdown, and other losses, such as material in process, even the hazards to the people and environment. The cost of minimal repair activities, which can be divided into two parts: before and after $N$ th halt at $t_{N}$, which is given by:

$$
C^{t c}(T)=\sum_{j=1}^{N} C_{j}^{c}+c_{j+1}^{c}(T)
$$

Where $C_{j}^{c}$ is the expected total cost of minimal repair and losses of the unscheduled breakdown in each preventive maintenance cycle before $t_{N}$, it can be obtained by:

$$
C_{j}^{c}=\sum_{i=1}^{M}\left[\left(c_{i}^{c}+l^{u}\right) \int_{t_{j-1}}^{t_{j}}(t) d t\right]
$$

The expectation of corrective maintenance cost and unscheduled breakdown losses in time horizon $\left[t_{N}, T\right]$ can be obtained by:

$$
C^{t c}(T)=\sum_{i=1}^{M}\left\{\left(c_{i}^{c}+l^{u}\right)\left[\sum_{j=1}^{N} \int_{t_{j-1}}^{t_{j}} \lambda_{i, j}(t) d t+\int_{t_{N}}^{T} \lambda_{i, N}(t) d t\right]\right\}
$$

Substituting (6) (7) into (5), the expected total cost of minimal repair and losses of unscheduled breakdown in time horizon $[0, T]$ can be represented by:

$C^{t c}(T)=\sum_{i=1}^{M}\left\{\left(c_{i}^{c}+l^{u}\right)\left[\sum_{j=1}^{N} \int_{t_{j-1}}^{t_{j}} \lambda_{i, j}(t) d t+\int_{t_{N}}^{T} \lambda_{i, N}(t) d t\right]\right\}$

In practice, the corrective maintenance cost per time is usually more than the one of preventive maintenance, because unscheduled maintenance job will disturb the spare parts plan and engineers work plan etc. Additionally, the losses of unscheduled breakdown are more than the scheduled halt also, because the production plan will be disturbed and the material in process will be wasted.

In this paper, the purpose is to obtain the minimal total maintenance cost of the system in a given finite time horizon through adjusting the thresholds of preventive maintenance and opportunistic maintenance, which can control the maintenance cost and actions times.

\subsection{Failure rate evolvement}

In section 3.1, the unit failure rate function is important in the expected maintenance cost functions of system, it is assumed that each unit's failure rate distribution function can be obtained, but due to imperfect maintenance actions, the actual function becomes very complex. In this paper, the effective age and the effective age renewal factor are introduced to describe the evolvement relationship of failure rate between two preventive maintenance cycles. 


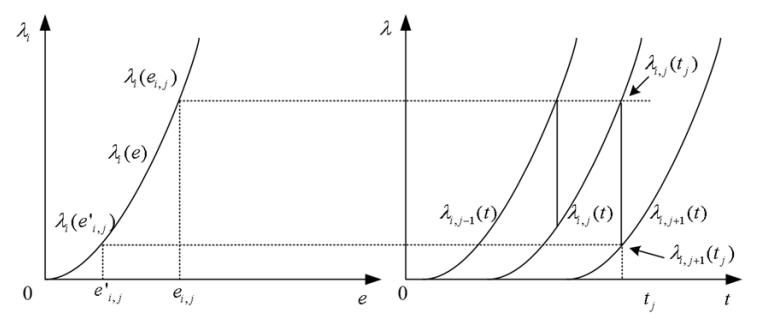

Figure 1 Relationship between effective age and actual age

The relationship between actual run-time and effective age of units is shown in Fig.1. At, before unitbeing taken maintenance action, the relationship between actual age and effective age can be written by:

$$
\lambda_{i, j}\left(t_{j}\right)=\lambda_{i}\left(e_{i, j}\right)
$$

And after being taken preventive or opportunistic maintenance, it is given by:

$$
\lambda_{i, j+1}\left(t_{j}\right)=\lambda_{i}\left(e_{i, j}^{\prime}\right)
$$

And in $j+1$ cycle, it can be represented by:

$$
\lambda_{i, j+1}(t)=\lambda_{i}\left(e_{i, j}^{\prime}+t-t_{j}\right), t \text { in }\left[t_{j+1}, t_{j+2}\right]
$$

Thus, for each unit, the change of failure rate function can be transformed into the change of failure rate of effective age. The relationship of effective age between before and after maintenance can be derived as follows.

According to assumption 6, the failure rate function of each unit does not change in each preventive maintenance cycle (the random failures in each preventive maintenance cycle can be corrected by minimal repair, and which does not change the failure rate function of each unit), therefore, the effective age of unit $i$ at $t_{j}$ before the $j$ th scheduled halt can be obtained by:

$$
e_{i, j}=e_{i, j-1}^{\prime}+\left(t_{j}-t_{j-1}\right)
$$

where $e_{i, j-1}^{\prime}$ is the effective age of unit $i$ after the $(j-1)$ th scheduled halt, according to various maintenance actions it can be represented by:

$$
e_{i, j}^{\prime}=\left\{\begin{array}{l}
e_{i, j}-\beta t_{i, j}^{s}, \text { repair } \\
0, \text { replacement } \\
e_{i, j}, \text { nomaintenance }
\end{array}\right.
$$

where $\beta$ is the effective age renewal factor of repair action, which represents the unit effective age renewal degree after repair. According to the assumption 2, the unit state after being repaired is not better than the one after the last maintenance activity, and the renewal quantum by repair is positively related with duration of continuous functioning without repair and replacement. $t_{i, j}^{s}$ can be obtained by:

$$
t_{i, j}^{s}=\left\{\begin{array}{l}
t_{i, j-1}^{s}+t_{j}-t_{j-1}, E_{i}\left(t_{j-1}\right) \geq E^{o} \\
t_{j}-t_{j-1}, E_{i}\left(t_{j-1}\right)<E^{o}
\end{array}\right.
$$

where $E_{i}\left(t_{j-1}\right) \geq E^{o}$ means that unit $i$ is taken preventive maintenance of opportunistic maintenance on at $t_{j-1}$.

And according to assumption 4, each unit is new at the beginning, then:

$$
\begin{aligned}
& e_{i, 0}=0 \\
& t_{i, 0}^{s}=0
\end{aligned}
$$

Then effective age of unit $i$ at any time in time horizon $[0, T]$ can be obtained by iterated operations with (9)-(16).

\subsection{Selection of maintenance actions}

In previous sections, the cost models have been built, and the changes of failure rate functions for various maintenance actions have been resolved by introduction of effective age and renewal factor. Then there is another important point, that is, how to make decision to select the alternative of suitable maintenance actions for the units that need be taken preventive maintenance actions when the system halts.

In this section, the expected failure rate reduced quantum per unit cost each maintenance cycle is used to make decision to select more economical maintenance action for those units which need to be taken maintenance. If unit $I$ is taken maintenance action at $t_{j}$, then its expectation of failure rate reduced quantum per maintenance cost is represented by:

$$
\begin{aligned}
\rho_{i, j} & =\left[\lambda_{i, j}\left(t_{j}\right)-\lambda_{i, j+1}\left(t_{j}\right)\right] / c_{i, j}\left(t_{j}\right) \\
& =\left[\lambda_{i}\left(e_{i, j}\right)-\lambda_{i}\left(e_{i, j}^{\prime}\right] / c_{i, j}\left(t_{j}\right)\right.
\end{aligned}
$$

At $t_{j}$, if $E_{i}\left(t_{j}\right) \geq E^{p}, i=1 \ldots, M$ then unit $i$ will be performed preventive maintenance, the expectation of failure rate reduced quantum per unit cost of unit $i$ by repair and replacement can be represented by:

$$
\begin{array}{r}
\rho_{i, j}^{p r}=\left[\lambda_{i}\left(e_{i, j}\right)-\lambda_{i}\left(e_{i, j}-\beta t_{i, j}^{s}\right)\right] /\left(c_{l}^{r}+l^{s}\right) \\
\rho_{i, j}^{p p}=\lambda_{i}\left(e_{i, j}\right) /\left[v_{i} \exp \left(-\int_{0}^{e_{i, j}} \lambda_{i}(t) d t\right)+c_{i}^{p}+l^{s}\right]
\end{array}
$$

If $E^{p}>E_{k}\left(t_{j}\right) \geq E^{o}, k=1, \ldots, M, k \neq i$ then unit $k$ will be performed opportunistic maintenance, and the expected failure rate reduced quantum per unit cost of unit $k$ by repair and replacement can be represented by:

$$
\rho_{k, j}^{o r}=\left[\lambda_{k}\left(e_{k, j}\right)-\lambda_{k}\left(e_{k, j}-\beta t_{k, j}^{s}\right)\right] / c_{l}^{r}
$$




$$
\rho_{k, j}^{o p}=\lambda_{k}\left(e_{k, j}\right) /\left[v_{k} \exp \left(-\int_{0}^{e_{k, j}} \lambda_{k}(t) d t\right)+c_{k}^{p}\right]
$$

If $\rho_{i}^{p p} \leq \rho_{i}^{p r}$,or $\rho_{k}^{o p} \leq \rho_{k}^{o r}$, then the repair action is more economical for units at $t_{j}$ and will be selected, else is replacement.

Minimal the total expected cost $C(T)$ in the time horizon $[0, T]$ can be obtained by changing the opportunistic maintenance threshold and preventive maintenance threshold, and then the preventive maintenance sequence scheduling can be obtained.

\subsection{Comparisons}

In the previous sections, the opportunistic maintenance policy was developed for multi-unit repairable systems with consideration of nonlinear deterioration process with age and introduction of imperfect maintenance. From a practical point of view, the opportunistic maintenance may not be efficient when the cost of scheduled halt less than wasted value of units which is replaced before being performed preventive maintenance. In other words, the savings must be larger than the losses. Then according to the assumption 7, the policy is efficient when the units parameters satisfy the following constraints:

$$
c_{i}^{r}+l^{s} \leq v_{i} \exp \left(-\int_{e_{i}^{-1}\left(E^{p}\right)}^{e_{i}^{-1}\left(E^{o}\right)} \lambda_{i}(t) d t\right)+c_{i}^{p}+l^{s} \leq c_{i}^{c}+l^{u}
$$

where $e_{i}^{-1}\left(E^{p}\right), e_{i}^{-1}\left(E^{o}\right)$,are the effective ages of unit $i$ when it respectively reaches the preventive maintenance threshold and opportunistic maintenance threshold.

In this paper, the units performance parameters can be obtained by historical data analysis, such as failure rate functions $\lambda_{i}(t)$ can obtained by statistical data of random failures of unit $i$, and the cost of each type of maintenance and the effective age renewal factor of repair can also be obtained through counting the average of maintenance records, and which will be constant in a finite time horizon since in usual, the technique of maintenance is not updated.

\section{Numerical example}

Consider the system with 4 units connected in series, and each unit failure probability density is follows the Weibull distribution. The performance and maintenance parameters are given in Table 1.

The failure rate function of Weibull distribution is

$$
\lambda(t)=\frac{\varphi}{\eta}\left(\frac{t}{\eta}\right)^{\varphi-1}
$$

Where $\varphi$ is the shape parameter, $\eta$ is the scale parameter.
Table 1 The performance and maintenance parameters.

\begin{tabular}{|c|c|c|c|c|c|c|}
\hline Units & $\varphi_{i}$ & $\eta_{i}$ & $v_{i}$ & $c_{i}^{p}$ & $c_{i}^{r}$ & $c_{i}^{c}$ \\
\hline 1 & 4 & 95 & 451 & 251 & 124 & 190 \\
\hline 2 & 3.2 & 140 & 290 & 174 & 129 & 160 \\
\hline 3 & 3.5 & 130 & 290 & 174 & 129 & 160 \\
\hline 4 & 3 & 110 & 335 & 262 & 135 & 145 \\
\hline
\end{tabular}

The downtime losses of the scheduled and unscheduled halts $l^{s}=220, l^{u}=400$.

Consider the time horizon $(0,1000]$, assume the effective age renewal factor of repair $\beta=0.75$, then the minimal total maintenance cost of the system $C^{*}(1000)=10431$, when the opportunistic maintenance threshold $E^{o^{*}}=57$, and the preventive maintenance threshold $E^{p^{*}}=35$. There are 13times of opportunistic repair, 3 time of opportunistic replacement, 11 times of preventive repair, 3 times of preventive replacement and 3 times of corrective maintenance. The cost of maintenance including opportunistic maintenance cost is 2290, preventive maintenance cost is 5359 and the corrective maintenance cost is 2782 .

Fig.2 shows the changing of times of each type of maintenance actions resulting from different opportunistic thresholds. It is obvious that the times of corrective maintenance and preventive maintenance increase with $E^{o}$ before the opportunistic maintenance threshold exceeds the preventive maintenance threshold. This is because before the opportunistic maintenance threshold is less than the preventive maintenance threshold, the maintenance opportunities triggered more when the interval is bigger between opportunistic maintenance threshold and the preventive maintenance threshold, which also means the excess maintenance that will cause the waste of maintenance cost.

Additionally, once the opportunistic threshold exceeds the preventive threshold, the maintenance opportunity will not be triggered any more. In this condition, more frequent preventive maintenances have to be performed on the units to keep the system state, and the random failures occur more frequently, which will cause more corrective maintenance cost and unscheduled breakdown losses.

Figs.3 and 4 show the change failure rate and expected total maintenance cost and the scheduled downtime losses of unit 1, it can be found the composite economic index is more rational and efficient than the single duration of running time index. This is because the expected total maintenance cost and the scheduled downtime losses is a comprehensive assessment of system state, maintenance cost and halts losses.

Fig.5 shows the change of times of repair and replacement with the effective age renewal factor of repair, it can be found that the times of repair decrease when the renew factors increase, indicating the times of 


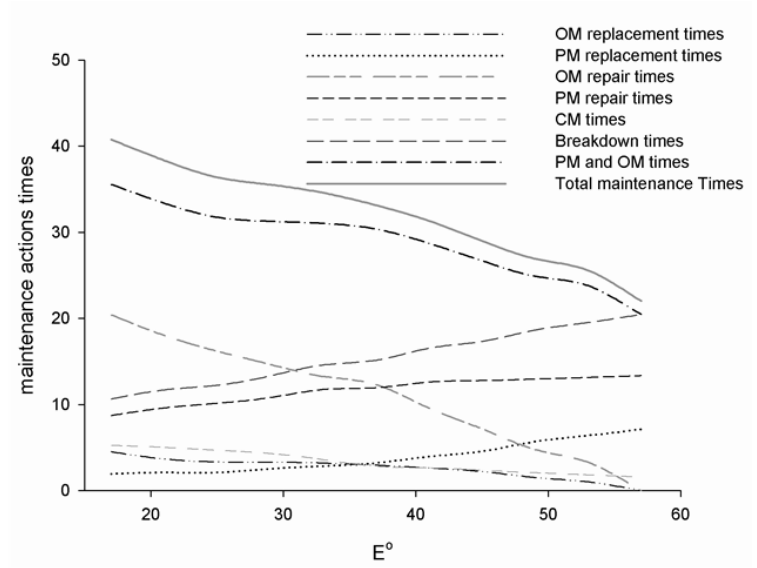

Figure 2 The times of various maintenance actions

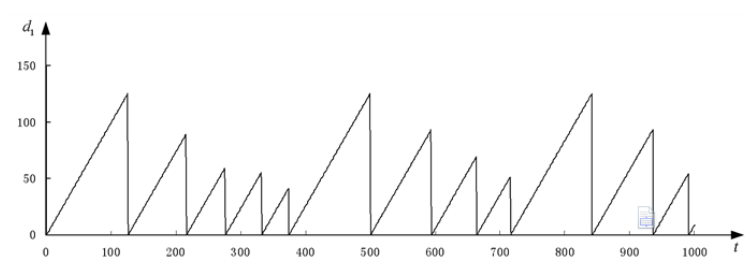

Figure 3 The change of duration time of unit 1 with time

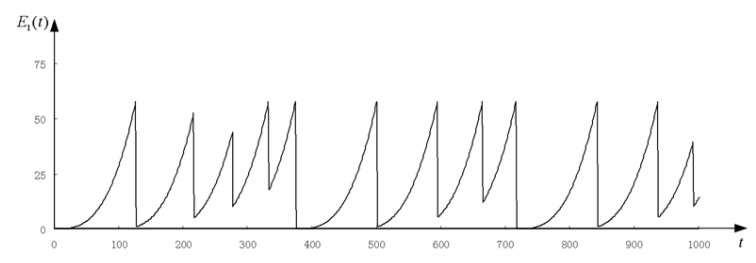

Figure 4 The change of expected maintenance cost of unit 1 with time

repair performed is positively correlated with efficiency of repair, which is also consistent with the actual situation.

\section{Conclusion}

In this paper, a new opportunistic maintenance policy is proposed for multi-unit serial repairable systems. The model considers that the unit deterioration process is nonlinear with age, and introduces of imperfect maintenance to be in line with actual. The model is optimized based on a comprehensive assessment model of system state, maintenance cost and halts losses. The

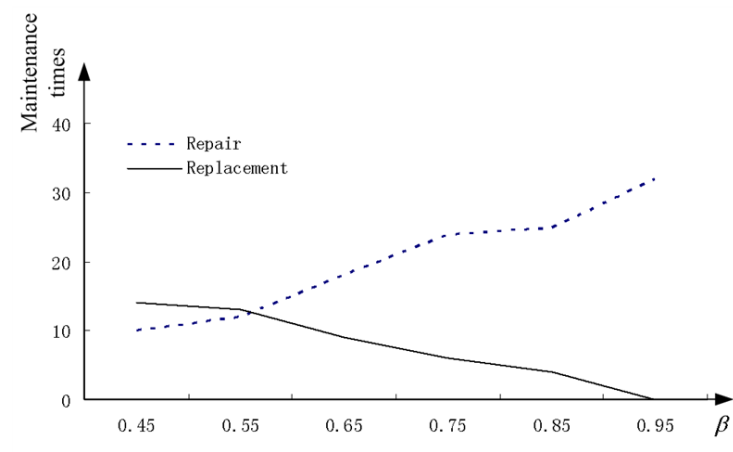

Figure 5 The change of times of repair and replacement $\beta$ with

maintenance cost model was constructed to count the total maintenance cost in finite time horizon. The maintenance effect is assessed through the expectation of failure rate reduced quantum per maintenance cost, which is used to make the decision of selection of maintenance actions.

The applicability of policy is also discussed. The policy in this paper can be further improved in some aspects:

(1)with consideration of the buffers between the units of the Series Production System in the future studies, in this situation, some maintenance opportunities maybe not occur, and the maintenance cost modes have to be improved, for the maintenance and repair time maybe not lead to make some loss.

(2)with consideration of serial-parallel production system, in this situation, the opportunistic maintenance model is more complex, because there are many possibility of the occurrence of the maintenance opportunities, the maintenance opportunity occurrence model has to be constructed and the maintenance actions selection model has to be improved.

(3)With consideration of expand the model optimization time horizon to the infinite time horizon from finite one.

\section{References}

[1] R. Radner and D.W. Jorgenson, Opportunistic replacement of a single part in the presence of several monitored parts, Management Science, 10 (1), $70-84$ (1963).

[2] M. Berg, Optimal replacement policies for two-unit machines with increasing running costs-I, Stochastic Processes and Applications, 5, 89-106 (1976).

[3] M.Berg, Comparison of age, block and failure replacement. IEEE transactions on Reliability, 27(1), 25-29, (1978).

[4] R.Dekker, and E.Smeitink, Preventive maintenance at opportunities of restricted duration, European Journal of Operational Research, 53, 46-63 (1991).

[5] R. Dekker, and M.C. Dijkstra, Opportunity-based age replacement: exponentially distributed times between opportunities, Naval Research Logistics, 39, 75-190 (1992). 
[6] X.Zheng, and N. Fard, A maintenance policy for repairable systems based on opportunisticfailure-rate tolerance, IEEE Transactions on Reliability, 40(2), 237-244 (1991).

[7] J. P.Stinson, B. M.Khumawala, Replacement of machines in a serially dependent multi-machine production system , International Journal of Production Research, 25(5), 677-688 (1987).

[8] J. Sarkis, J. Semple, An optimal multi-machine replacement policy in a serially dependent production system, International Journal of Production Research, 32(11), 2657-2667 (1994).

[9] X. Zheng , All opportunity-triggered replacement policy for multiple-unit systems, IEEE Transactions on Reliability, 44(4), 848-652 (1995).

[10] J. D. Diederik, and A. M. Jan, Coordinated conditionbased repair strategies for components of a multi-component maintenance system with discounts, European Journal of Operational Research, 98(1), 52-63 (1997).

[11] M. Savsar, M. Aldaihani,, A Stochastic Model for Analysis of Manufacturing Modules, Applied Mathematics \& Information Sciences, 9(6), 587-600 (2012).

[12] H. Pham, and H. Wang, Imperfect maintenance, Naval Research Logistics, 47(3), 223-239 (2000).

[13] A.N. Rao, and B. Bhadury, Opportunistic maintenance of multi-system system: a case study, Quality and Reliability Engineering International, 16, 487-500 (2000).

[14] T. Satow, and S. Osaki, Opportunity-based age replacement with different intensity rates, Mathematical and computer modeling, 38, 1419-1426 (2003).

[15] X. Zhou, and L.Xi, J. Lee, A dynamic opportunistic maintenance policy for continuously monitored systems, Journal of Quality in Maintenance Engineering, 12(3), 294305 (2006).

[16] E. Levrat, E. Thomas, and B. Iung,. Odds-based decisionmaking tool for opportunistic production-maintenance synchronization, International Journal of Production Research, 46:19, 5263-5287 (2008).

[17] X. Zhou, L. Xi and J. Lee. Opportunistic preventive maintenance scheduling for a multi-unit series system based on dynamic programming, International Journal of Production Economics, 118(2), 361-366 (2009).

[18] X. Zhou, Z. Lu, L. Xi and J. Lee. Opportunistic preventive maintenance optimization for multi-unit series systems with combing multi-preventive maintenance techniques, Journal Of Shanghai Jiaotong University (SCIENCE), 15(5), 513-518 (2010).

[19] B. Tim, D. Isha, M. Isaac, and Z. Athena, The signal model: A model for competing risks of opportunistic maintenance, European Journal of Operational Research, 214(3), 665-673 (2011).

[20] R.E. Barlow, L.C. Hunter, Optimum preventive maintenance policies, Operations Research, 8, 90-100 (1960).

[21] H. Pham, and H. Wang, Imperfect maintenance, European Journal of Operational Research, 94, 425-438 (1996).

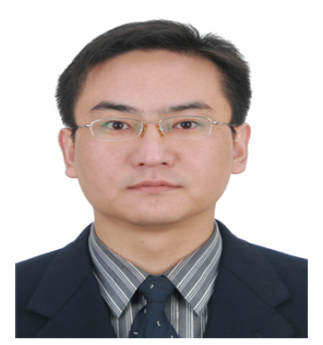

Wenrui Hou received the MS degree in Management science and Engineering from Shanghai Jiaotong University in 2005. He is currently a $\mathrm{PhD}$ Candidate Supervisor in Shanghai Jiaotong University. His research interests are in the areas of opportunistic maintenance and information systems.

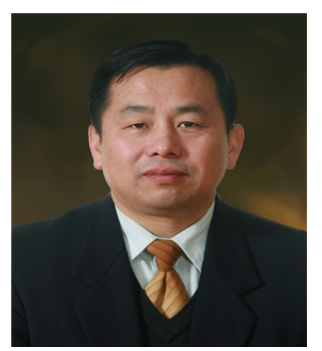

Zuhua Jiang received the MS degree in Mechanical Engineering from Harbin Engineering University in 1990, and the PhD degree in Mechanical Engineering from the Shanghai Jiaotong University in 1998. He is currently a professor in Shanghai Jiaotong University. His research interests are in the areas of opportunistic maintenance, knowledge management, and information systems. 